\title{
Auger processes in InAs self-assembled quantum dots
}

\author{
P. P. Paskov ${ }^{\text {a }}$, P. O. Holtz ${ }^{\text {a }}$ S. Wongmanerod ${ }^{a}$, B. Monemar ${ }^{\text {a }}$, J. M.Garcia ${ }^{\text {b }}$, W. V. \\ Schoenfeld ${ }^{b}$ and P. M. Petroff ${ }^{b}$ \\ ${ }^{a}$ Department of Physics and Measurement Technology, Linköping University, S-581 83 \\ Linköping, Sweden \\ ${ }^{\mathrm{b}}$ Materials Department, University of California, Santa Barbara, CA 93106, USA
}

\begin{abstract}
An experimental evidence of Auger-like excitation processes in InAs/GaAs quantum dots is demonstrated. Photoluminescence spectra of resonantly excited dots exhibit a rich satellite structure below the ground-state emission band. The energy position and the intensity distribution of the satellites are analyzed and an interpretation of the satellites as due to shakeup processes of the interacting carriers in the higher quantum dot states is suggested.
\end{abstract}

The potential of quantum dot (QD) structures for optoelectronic device applications has been frequently questioned due to the suppression of the carrier relaxation rate in zero-dimensional systems [1]. The discrete nature of the density-of-states dispersion in QDs prevents the effective carrier relaxation unless the electron level spacing is smaller than a few meV to favour the LA-phonon scattering or equals the typical LO-phonon energies. This effect, referred to as the "phonon bottleneck" , should result in a considerable reduction of the luminescence efficiency and should also constitute a significant limitation of the performance for QD-based devices. The role of phonons in the relaxation of the carriers in self-assembled InGaAs/GaAs QDs have been studied by resonant excited photoluminescence (PL) and radiative lifetime measurements of excited states [2 and 3].The phonon bottleneck problem could be removed or at least reduced if the carrier transfer to lower states could occur via Auger processes [4, 5 and 6]. Experimentally, Auger processes can be demonstrated by means of satellite spectroscopy. In this case, the photo-excited electron-hole pair interacts with other carriers and as result an excitation of the additional carriers in higher states takes place. These shake-up processes can be monitored in the PL spectra as satellites which are red-shifted relatively the principal electron-hole pair recombination. The satellite spectroscopy has been a widely used method to study Auger-like processes in semiconductor quantum wells (QWs), but the final state for the interacting carriers varies for different shake-up processes, e.g., a higher Landau level for an electron gas or a negatively charged exciton in a magnetic field [7 and 8] and a higher subband for a two-particle transition [9]. The prerequisite 
for all these processes is the localization of the involved particles. In this respect, the situation should be even more favourable in QDs. We report here satellite spectroscopy experiments on self-assembled InAs/GaAs QDs.

The QD samples used in this study were grown by molecular beam epitaxy on top of an $\mathrm{n}^{+}$doped GaAs ( 100 ) substrate [10]. The dots were formed by depositing $1.7 \ln A$ s monolayers at $T_{g}=530^{\circ} \mathrm{C}$. A growth interruption of $30 \mathrm{~s}$ was used to narrow down the size distribution. Then the dots were overgrown at $T_{g}=530^{\circ} \mathrm{C}$ with a thin GaAs cap of a thickness adjusted between 1 and $7.5 \mathrm{~nm}$ followed by a second $30 \mathrm{~s}$ growth interruption and a final capping with GaAs. Atomic force microscopy (AFM) after such a growth procedure revealed well-defined lensshaped islands with narrow size distribution. With a decrease of the capping layer thickness, the height of the InAs islands is reduced from 10 to $1.5-2.5 \mathrm{~nm}$, while their lateral dimensions typically range $60-80 \mathrm{~nm}$ [10].

The non-resonant PL spectra of the QD sample at three different excitation intensities are shown in Fig. 1. At low excitation intensity only the QD ground-state emission at $1.303 \mathrm{eV}$, is observed. With an increase of the laser intensity the ground-state emission saturates and two additional peaks attributed to the recombination from QD excited states appear at 1.348 and $1.385 \mathrm{eV}$. The calculations of the electronic structure of similar lens-shaped InAs/GaAs QDs predict several confined states for electrons and holes [11]. The ground states (s-like shell) have a magnetic momentum $m=0$ and can be occupied by only two carriers. The next higher p-like $(m= \pm 1)$ and d-like $(m=0, \pm 2)$ shells can hold up to four and six carriers, respectively. Due to the selection rules, only transitions between states with an equal magnetic momentum are possible. Then we identify the peaks at 1.348 and $1.385 \mathrm{eV}$ as the electron-hole recombination from the $p$ - and d-like shells, respectively. Such an interpretation is confirmed by magneto-PL measurements shown in Fig. 2. In a magnetic field, the second PL peak splits into two bands as expected for a transition between states with $p$-like character, while the ground-state emission exhibits only a diamagnetic shift of $\approx 10 \mu \mathrm{eV} / \mathrm{T}^{2}$.

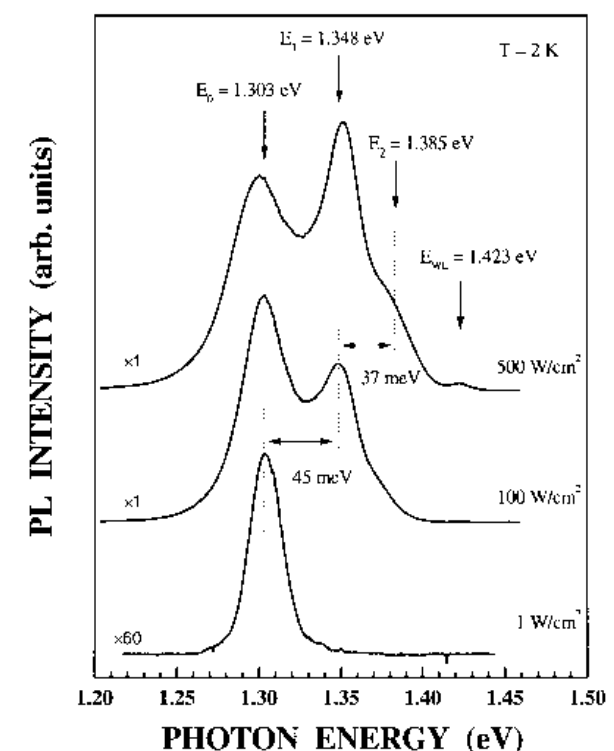

Fig. 1. Intensity-dependent PL spectra of the $\mathrm{QD}$ sample excited with an $\mathrm{Ar}^{+}$-laser. The arrows mark the position of the ground-state $\left(E_{0}\right)$, the excited states $\left(E_{1}, E_{2}\right)$, and the wetting layer $\left(E_{W L}\right)$ emissions. 


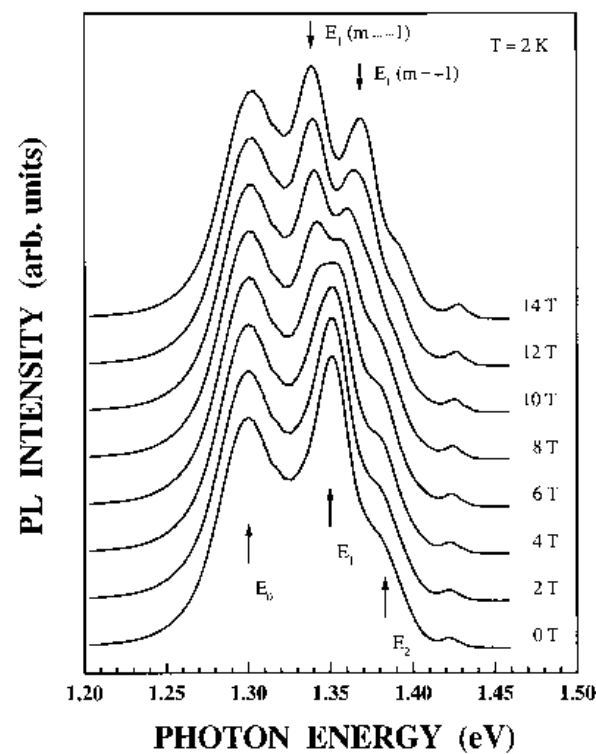

Fig. 2. Magneto-PL spectra measured at an excitation intensity of $500 \mathrm{~W} / \mathrm{cm}^{2}$ and different magnetic fields applied parallel to the growth direction.

The emission of our specially designed samples is at a higher energy than is observed for the standard InAs/GaAs self-assembled QDs. The blue shift of the spectrum enables us to carry out resonant PL spectroscopy by means of a tunable $\mathrm{Ti}$ : Sapphire laser. As long as the excitation is above the ground-state emission, the PL spectrum is dominated by the peak at $1.303 \mathrm{eV}$, but when the laser energy is further down-shifted, novel satellites are observed at lower energies. Fig. 3 shows the PL spectrum with an excitation at the maximum of the ground-state emission. There are three distinguishable satellites, labelled $S_{1}, S_{1}$ and $S_{2}$. The two sharp lines observed at 33.8 and $36.6 \mathrm{meV}$ below the laser energy correspond to the Raman modes of bulk GaAs TO and LO phonons. With a decreasing excitation energy, the satellites appear at a slightly varying energy separation from the laser energy. This is clearly illustrated in Fig. 4, where the satellite spectra are plotted with the laser energy as a reference energy. There are several notable observations to be extracted from Fig. 4. As the excitation energy is decreased, the overall intensity level is reduced, since there will be successively fewer dots resonantly excited in this region. There is a general trend towards increasing satellite energies, defined as the energy displacement between the laser excitation and the satellite peak position, with decreasing laser excitation energy. Finally, the satellite intensity distribution is changed in such a way that high-energy satellites are enhanced with decreasing excitation energy. 


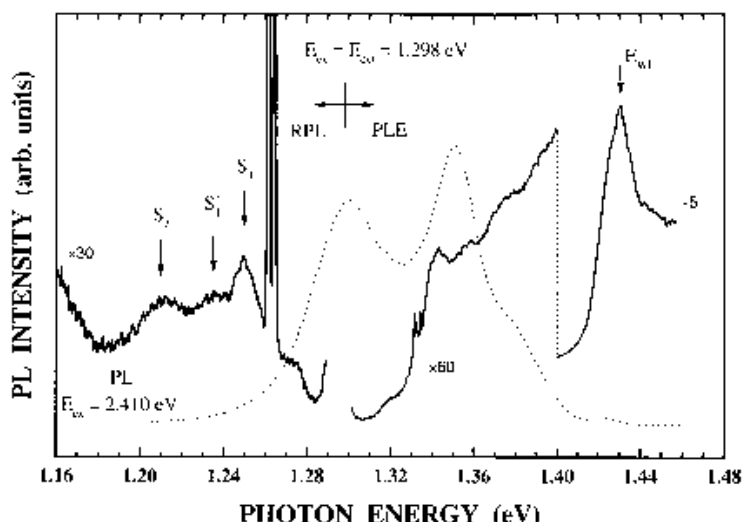

PIOTON ENERGY ( $\mathrm{NV}$

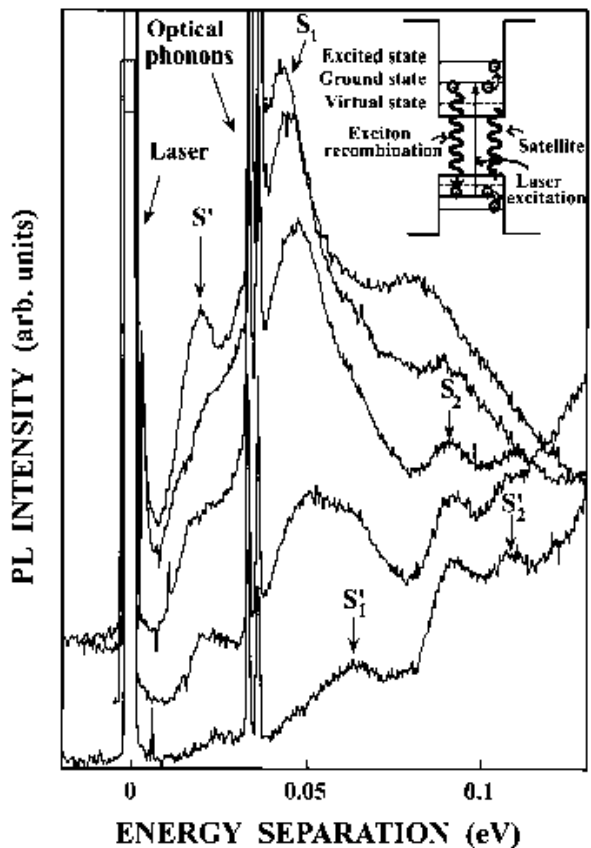

Fig. 3. Resonant $\mathrm{PL}$ spectrum (RPL) of the QD sample excited at $E_{e x}=1.298$ eV and the corresponding PLE spectrum detected at the same energy. The
Fig. 4. RPL spectra as a function of energy separation from the laser energy. The excitation energy is successively downshifted from $1.3 \mathrm{eV}$ (top spectrum) to 1.26 $\mathrm{eV}$ (bottom spectra) by a step of $10 \mathrm{meV}$. The inset shows a schematic description of the shake-up process.

As can be seen in Fig. 3 the satellite spectrum exhibits a nearly mirror-like symmetry with the corresponding PL excitation (PLE) spectrum. This fact together with the excitation energy dependence of the satellite position implies an electronic rather than phonon-related origin of the satellites. The interpretation of the satellite spectra is based on an Auger-like process, in analogy with the earlier reported satellites in QWs [9]. The photo-excited electron-hole pair can interact with other carriers localized in the dot to form biexcitons, charged excitons and/or multiexciton complexes [12 and 13]. At the electron-hole recombination, the additional carriers are normally left in their ground state, however, there is non-zero probability that these carriers are excited into an empty higher state. This will result in a satellite, which is red-shifted relatively the principal electron-hole pair recombination with an energy separation corresponding to the shake-up energy (see the inset in Fig. 4). When an electron-hole pair is shaken up into the first or the second excited state, the satellite energies should be similar to the energy splitting between the emission peaks observed in the non-resonant PL. Infact, the 
energies of the two predominant satellites $S_{1}$ and $S_{2}, 44-52$ and $84-92 \mathrm{meV}$, are in a good agreement with the energy separations $E_{1}-E_{0}=45 \mathrm{meV}$ and $E_{2}-E_{0}=82 \mathrm{meV}$. The excess in satellite energy can be attributed to the biexciton binding energy of 2-4 meV [13]. The satellites $S_{1}{ }^{\prime}$ and $S_{2}{ }^{\prime}$ appear at an energy of ${ }^{13-15} \mathrm{meV}$ higher than the satellites $S_{1}$ and $S_{2}$, respectively. This energy separation is practically independent on the excitation energy implying some kind of pairing between these satellites. Moreover, with decreasing excitation energy, the satellites $S_{1}{ }^{\prime}$ and $S_{2}{ }^{\prime}$ gain intensity relatively the satellites $S_{1}$ and $S_{2}$. The difference in energies of the paired satellites is proposed to be due to the Coulomb blockade effect when the electrons are shaken up into a partly filled shell. Due to the smaller interlevel spacing, the hole relaxation is much faster and an excess of electrons in the higher states can be expected. In this case, the shake-up energy should overcome the electron-electron interaction and will result in a red-shifted satellite. The estimations of the electron addition energies for $p$ - and $d$ shell yield a value of 12-14 meV [14], consistent with the observed energy separation of 13$15 \mathrm{meV}$. Summarising the above consideration, we interpret the satellites $S_{1} / S_{1}{ }^{\prime}$ and $S_{2} / S_{2}{ }^{\prime}$ as a biexciton being the initial state and in the final state one exciton recombines as a satellite, while the second exciton is shaken up into the first or the second excited state, respectively. The origin of the weak satellite $S^{\prime}$ is currently not fully understood, but one possible explanation is a recombination of a negatively charged exciton and a shake-up of the second electron into the p-like electron shell. The energy of $\mathrm{S}^{\prime}(21-25 \mathrm{meV})$ is very close to the electron single-particle energy of $24 \mathrm{meV}$ as estimated from the simple parabolic potential model [14].

The increase of the satellite energies with decreasing excitation energy is explained in terms of a varying QD size. When the laser energy is down-shifted from the maximum of the groundstate emission, the thicker dots are excited. These dots have a smaller lateral dimension as shown by AFM [10] and consequently, a larger intersubband spacing. The trend of the satellite energies is consistent with the results from PL measurements on other QD samples from the same growth series. The PL spectrum of the samples with a capping layer of $7.5 \mathrm{~nm}$ is shifted towards lower energies with the ground-state emission peaking at $1.16 \mathrm{eV}$, while the spacing between the emission bands increases to about $72 \mathrm{meV}$.

In conclusion, satellite spectroscopy has been performed to study Auger-like processes in InAs/GaAs self-assembled QDs. The rich satellite structure observed with a resonant excitation of the low-energy part of the PL emission is interpreted as being to shake-up processes of interacting carriers in the QD excited states.

\section{References}

1. H. Benisty, C.M. Sotomayor-Torres and C. Weisbuch. Phys. Rev. B 44 (1991), p. 10945.

2. S. Farfad, R. Leon, D. Leonard, J.L. Merz and P.M. Petroff. Phys. Rev. B 52 (1995), p. 5752.

3. S. Raymond, S. Farfad, P.J. Poole, A. Wojs, P. Hawrylak, C. Gould, A. Sachrajda, S. Charbonneau, D. Leonard, R. Leon, P.M. Petroff and J.L. Merz. Superlat. Microstruc. 21 (1997), p. 541.

4. U. Bockelmann and T. Egeler. Phys. Rev. B 46 (1992), p. 15574. 
5. A.V. Uskov, F. Adler, H. Schweizer and M.H. Pilkuhn. J. Appl. Phys. 81 (1997), p. 7895.

6. R. Ferreira and G. Bastard. Appl. Phys. Lett. 74 (1999), p. 2818.

7. K.J. Nash, M.S. Skolnick, M.K. Saker and S.J. Bass. Phys. Rev. Lett. 70 (1993), p. 3115.

8. G. Finkelstein, H. Shtrikman and I. Bar-Joseph. Phys. Rev. B 53 (1996), p. 12593.

9. P.O. Holtz, Q.X. Zhao, B. Monemar, M. Sundaram, J.L. Merz and A.C. Gossard. Phys. Rev. B 50 (1994), p. 4439.

10. J.M. Garcia, T. Mankad, P.O. Holtz and P.J.Wellman, P.M. Petroff. Appl. Phys. Lett. 72 (1998), p. 3172.

11. A. Wojs, P. Hawrylak, S. Fafard and L Jacak. Phys. Rev. B 54 (1996), p. 5604.

12. E. Dekel, D. Gershoni, E. Ehrenfreund, D. Spektor, L.M. Garcia and P.M. Petroff. Phys. Rev. Lett. 80 (1998), p. 4991.

13. M. Bayer, T. Gutbrod, A. Forchel, V.D. Kulakovskii, A. Gorbunov, M. Michel, R. Steffen and K.H. Wang. Phys. Rev. B 58 (1998), p. 4740.

14. R.J. Warburton, B.T. Miller, C.D. Durr, C. Bödefeld, K. Karrai, J.P. Kothaus, G. Medeiros-Ribeiro, P.M. Petroff and S. Huant. Phys. Rev. B 58 (1998), p. 16221. 